\title{
Home Security using Face Recognition Technology
}

\author{
Telugu Maddileti, G. Shriphad Rao, Vaddemani Sai Madhav, Ganti Sharan
}

\begin{abstract}
Face is the easiest way to penetrate each other's personal identity. Face recognition is a method of personal identification using the personal characteristics of an individual to decide the identification of a person. The method of human face recognition consists basically of two levels, namely face detection and face recognition. There are three types of methods that are currently popular in the developed face recognition pattern, those are Eigen faces algorithm, Fisher faces algorithm and CNN neural network for face recognition
\end{abstract}

Keywords: Face recognition, Face-detection, Eigen-faces, Fisher-faces, CNN, neural network, Residual network.

\section{INTRODUCTION}

The act of recognizing a known object as a known or unknown face is facial recognition. The topic of facial recognition is often stressed with the difficulty of face recognition [1],[2],[3].On the other hand, face recognition is to apply a face database to validate this input face to determine whether a "face" belongs to an acquaintance or an unknown personas said by [4].

This study discussed about the three trending methods in the face recognition. Those are eigen faces, fishers face algorithms and ResNet with 29 convolution layers. Once the face is recognized as an owner of the house, a secure token is sent to the cloud and the Node MCU collects the token from the cloud and validates the token, if the token is correct then node MCU sends a signal to the servo motor to open the door for owner.

This project is inspired by Davis king and Adam Geitgey, who has made it publicly accessible. This study uses pre-trained neural network from dlib, which will be discussed in upcoming chapters.

Revised Manuscript Received on December 30, 2019.

* Correspondence Author

Telugu Maddileti, Assistant Professor, ECM Department, Sreenidhi Institute of Science and Technology, Ghatekesar, Telangana-501301. E-mail:madhu14283@gmail.com.

G.Shriphad Rao, ECM Department, Sreenidhi Institute of Science and Technology, Ghatekesar, Telangana-501301

E-mail: shriphadnike@gmail.com

Vaddemani Sai Madhav, ECM Department, Sreenidhi Institute of Science and Technology, Ghatekesar, Telangana-501301

E-mail: vaddemanimadhav@gmail.com

Ganti Sharan,, ECM Department, Sreenidhi Institute of Science and Technology, Ghatekesar, Telangana-501301

E-mail:gantisharan6639@gmail.com

(C) The Authors. Published by Blue Eyes Intelligence Engineering and Sciences Publication (BEIESP). This is an open access article under the CC BY-NC-ND license (http://creativecommons.org/licenses/by-nc-nd/4.0/)

\section{LITERATURE SURVEY}

This paper discusses about the two currently popular face recognition techniques those are:

1. Eigen faces

2. Fisher faces

\section{A. Existing System}

In the Existing system, the face is detected or recognized using older method such as Eigen faces and Fisher face. Both approaches employ techniques which includes principal aspect analysis (PCA) and Linear Discriminant analysis (LDA) to minimize dimensions [5].

\section{B. Eigenfaces}

Eigenfaces is a type for numerical approach-based face recognition. This PCA-based algorithm reduces the quantity of dimensions while the maximum crucial records are retained. This algorithm's goal is to eliminate the main components that most affect the variation of the image [6].

Pre-processing of the image into grey scale is required for the training of the data. PCA considers every pixel of an image is one dimension. The following are steps are used in this method:
1. Convert the images to matrix
2. Adjust the matrix to a vector
3. Calculate the vector's mean
4. Remove the mean from the vector
5. Calculate a matrix of covariances
6. Calculate eigen values and vector

These steps are followed in the training of the data, when an unknown face is given as a testing data to this model, it finds outs the shortest Euclidean distance between the unknown data and training data, whichever has the shortest distance is predicted.
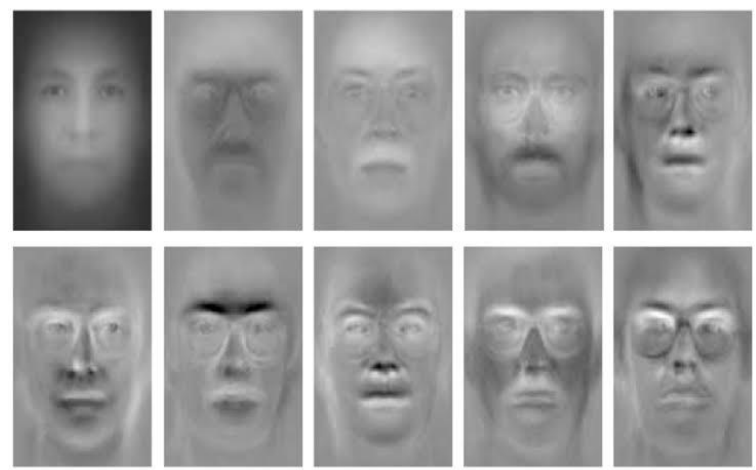

Figure-2.1.1: Eigen faces 


\section{Home Security using Face Recognition Technology}

Although this algorithm recognizes the faces but it has a lot of limitations, some of them are described below:

1. This method is scale sensitive that is it requires pre-processing of the image for scale normalization.

2. The accuracy decreasing for different poses of the testing image.

3. It requires plain or uniform background.

4. Eigen faces do not differentiate between two pictures from different classes during the training

\section{Fisherfaces}

Fisherfaces are just like Eigenface, the main distinction among them is the Linear Discriminant evaluation (LDA) approach used in Fisherfaces. As mentioned in [7] and we took [8] as a reference.

As discussed that Eigen faces do not make the difference during the training between two pictures from different classes from above, due to this whole average is affected.

Fisherface algorithm overcomes this hassle with the aid of the use of LDA for lowering the scale of the image.

Within class scatter, the LDA maximizes the class dispersion ratio to that, so it works better than PCA. The intention is to lessen the variance inside a category in comparison to the variant among classes, but an important procedure will also be the average per class. The average is calculated by given formula below:

$$
\Psi_{c_{i}}=\frac{1}{q_{i}} \sum_{k=1}^{q_{i}} \Gamma_{k}
$$

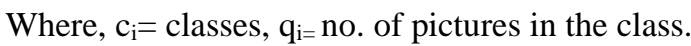

Unlike Eigenfaces, subtract the average of the corresponding class from each vector

$\Phi_{i}=\Gamma_{i}-\Psi_{c_{i}}$

Then the scatter matrices are calculated, and intra-class scatter is represented as $S_{w}$, further inter-magnificence scatter is represented as $\mathrm{Sb}$. After that, the goal is to discover a projection ' $\mathrm{W}$ ' that maximizes the optimization criteria of Fisherfaces.

$W_{o p t}=\arg \max _{w}=\frac{\left|w^{T} s_{b} W\right|}{\left|w^{T} s_{w} W\right|}$

Then the process is similar to PCA, the training image projection will be compared to the test image projection.

The prediction of the algorithm will be the class of the image with the smallest distance.

\section{Eigen Faces Vs Fisherfaces}

Eigenface attempts to maximize variation. They usually get a decent face model with PCA, but Fisherface wants the mean distance of different classes to be maximized while minimizing the variance within the class. They are faced with models that are more useful for discrimination [8].

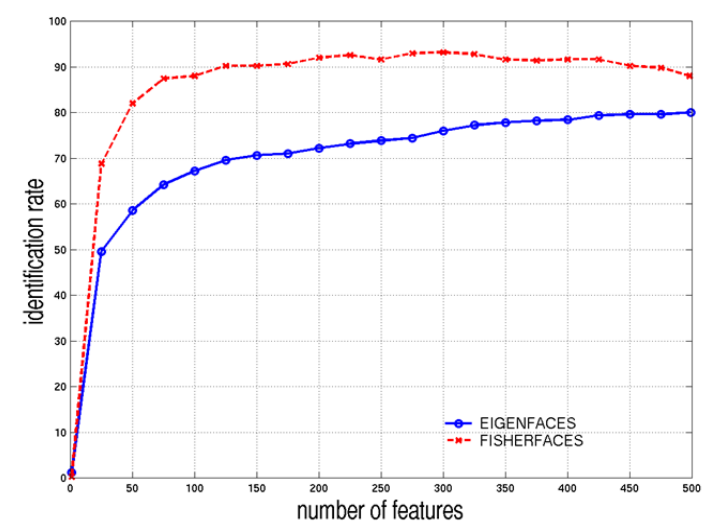

Figure 2.1.3: Comparison between Eigenface and Fisherface

As the below figure clearly tells that Fisherfaces algorithm is better than Eigen faces algorithm.

Although Fisherface has better accuracy that Eigenfaces but it has some limitations some of them are given below:

1. Fisherface is more complex than Eigenface.

2. Fisherfaces do not solve the issue of pose variations.

3 . The accuracy is not always stable.

4. Accuracy in real-time video is not pleasant.

\section{PROPOSED SYSTEM}

As discussed in the above sections, it is not ideal to use Eigenfaces or Fisherfaces for face recognition because both of the algorithm does not work with different poses, different angles of the same face and different photos of the same person, the accuracy is not up to the mark. Hence the paper proposes another method to recognize the face.

\section{A. Deep Neural networks}

Deep learning comes under machine learning that has networks that can learn from unstructured or unlabeled data without supervision. It has a couple of layers those are input layers, hidden layers and output layers. The network is going through the layers to measure each layer's likelihood. For more information please refer [9].

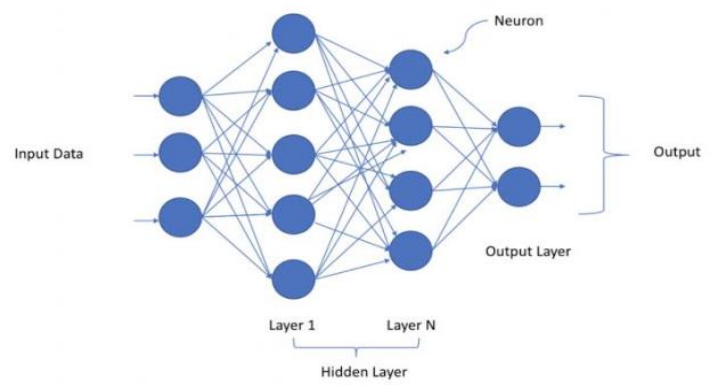

Figure 3.1: Deep neural network

\section{B. Convolutional Neural networks (CNN)}

Convolutional neural networks enable computers to see, meaning that $\mathrm{CNN}$ is used to recognize images by transforming the original image into class scores through layers. CNN allows us to extract all the features from the input image [10]. This process is generally done by two things those are feature learning and classification. 


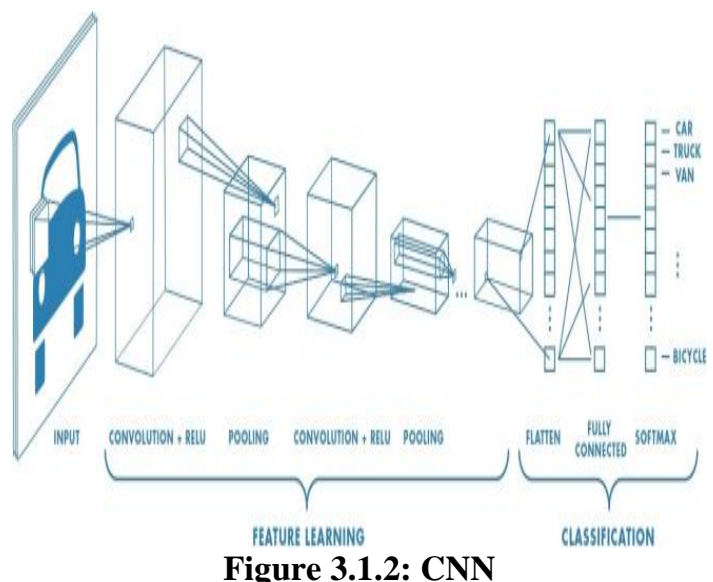

\section{Residual Networks (ResNet)}

The famous known vanishing gradient is one of the problems that ResNet solve. When the network it too deep then the gradient will become zero, this problem is overcome by ResNet. When a network is built with too many layers then the training error increases, but while case of ResNet deeper layers does not affect the training error.

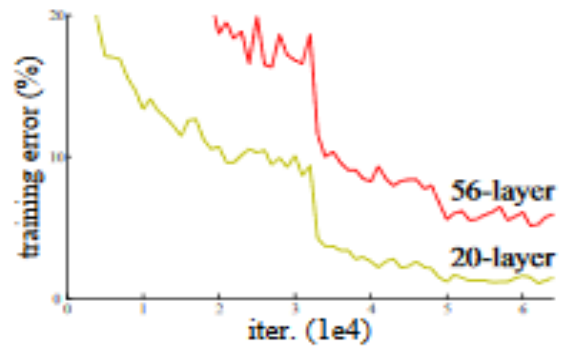

Figure 3.1.3(a): Training error of plain network

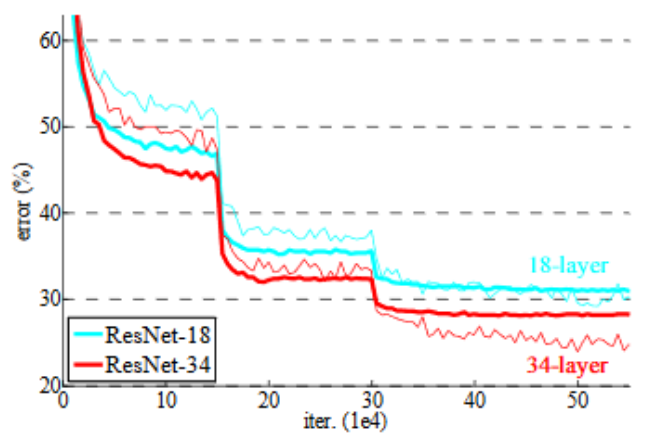

Figure 3.1.3(b): Training errors of ResNet

Deeper layers can be added but the overall error won't be affected, because it contains residual network which steps the layer [11].

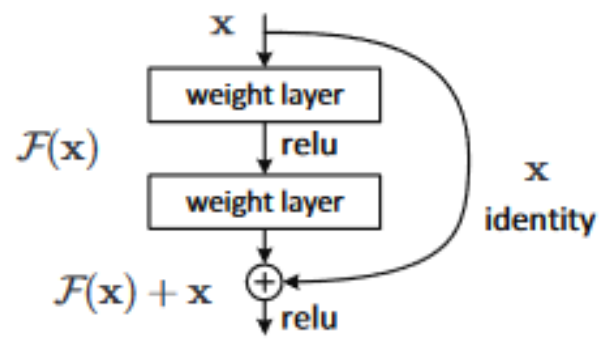

Figure 3.1.3(c): Residual Network

\section{Face Recognition}

This study uses a pre-trained model which is a 29-layer Resnet network dlib (library). This design is a Resnet-34 with few layers and a reduction of half of the number of filters consistent with layeras said by [12]. This model was used for face recognition.

dlib_face_recognition_resnet_model_v1.dat:

The network was trained with 3 million images of face, the data was taken from face scrub and VGG datasets. This model maps human faces into $128 \mathrm{D}$ vectors in which images of the same person are mapped near to each other and images of different individuals are mapped far apart.The resulting model obtains anaccuracy of $99.38 \%$ with a deviation of 0.00272732 on the LFW benchmark.

shape_predictor_5_face_landmarks.dat:

This is a template of 5 points land marking which distinguishes the eyes ' corners and the nose's edge. It is educated on the landmark dataset, which consists of 7198 faces. Predicts the face's shape that is basically specifies how the shape is positioned in the picture.

\section{E. Face Recognition Block diagram}

The working of the face recognition is described below [13].

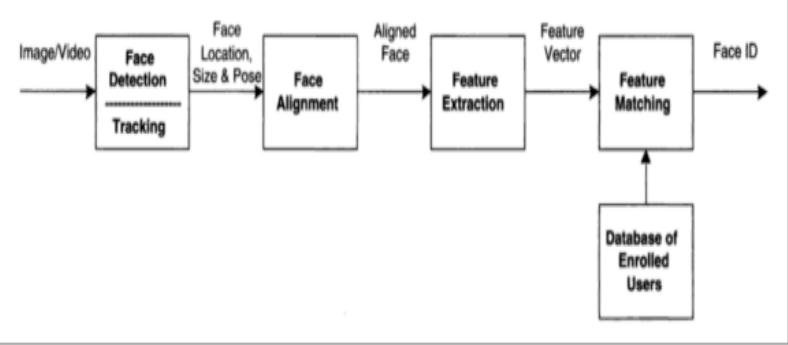

Figure 3.2.1: Block Diagram

Face recognition is actually a sequence of several related steps:

1. The work done by the first block is to get the face from the input image by using dlib's frontal_face_detector, the returns a face detector while that is used to get the faces from the input image or video.

2. In the second block, we useshape_predictor_5_face_landmarksto predict the shape of the face detected by the first block.

3. In the third block, we use the trained neural network model that is dlib_face_recognition_resnet_model_v1 it produces $128 \mathrm{D}$ vector of the aligned face from the second block. This $128 \mathrm{D}$ vector describes the face.

4. In the face matching block, the vector is compared to the known faces' vectors, if it is matched then predict as owner of the house.

Thanks to [11] who has made a library Face_recognition which made it lot easier to do all the above steps with just by calling the appropriate function when needed.

If recognized as an owner:

When an owner of the house comes in front of the camera, the above process takes place and when the face is recognized a security token is send to the cloud.

If not recognized as an owner:

When an unknown person before the lens, as usually the face recognition steps takes place and then saves the unknown person in the unknown's folder so that the owner of the house can see all the unknown faces who tried to enter the house. 


\section{Home Security using Face Recognition Technology}

\section{F. Work with Node MCU}

As discussed earlier, whenever an owner appears in front of the camera a secure token is sent to cloud, here the working of the NodeMCU takes place. NodeMCU contains a Wi-Fi module which is esp8266, this module is used to connect to the internet. For more information please refer to [12]

The working of NodeMCU is simple, when it is connected to the web; it tries to retrieve a value from the cloud which was used for face recognition. Then the value received from the cloud is evaluated, if verified as true then the NodeMCU send a signal to the servo motor to turn 90 degrees, so the lock to the door is opens. The whole code for NodeMCU is written Arduino IDE.

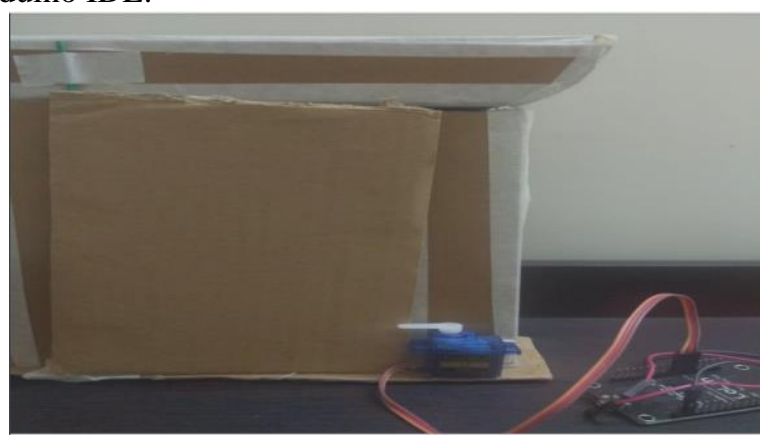

Figure 3.3.1: Door Locked

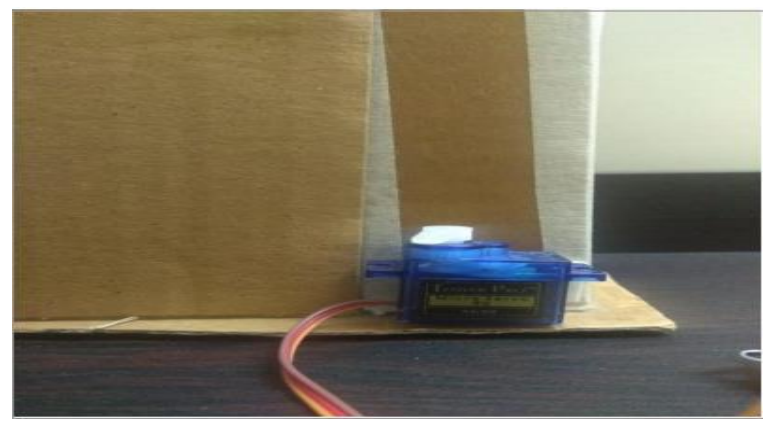

Figure 4.2: Door Unlocked

\section{RESUlTs}

Both the existing and proposed system is taken into consideration, so that we can compare the results to each other.

\begin{tabular}{|c|c|c|c|}
\hline $\begin{array}{c}\text { Type of } \\
\text { recog } \\
\text { nition }\end{array}$ & $\begin{array}{r}\text { Eigenfaces } \\
\text { algo } \\
\text { rith } \\
\text { m }\end{array}$ & $\begin{array}{r}\text { Fisherfaces } \\
\text { algori } \\
\text { thm }\end{array}$ & $\begin{array}{r}\text { Neural net } \\
\text { wo } \\
\text { rk }\end{array}$ \\
\hline Picture & $\checkmark$ & $\checkmark$ & $\checkmark$ \\
\hline $\begin{array}{c}\text { Real-time } \\
\text { video }\end{array}$ & Low accuracy & $\begin{array}{c}\text { Medium } \\
\text { accuracy }\end{array}$ & $\begin{array}{l}\text { Ideal } \\
\text { accuracy }\end{array}$ \\
\hline $\begin{array}{c}\text { Different } \\
\text { lighti } \\
\text { ng }\end{array}$ & $\times$ & $\checkmark$ & $\checkmark$ \\
\hline $\begin{array}{c}\text { Different } \\
\text { angle } \\
\text { s }\end{array}$ & $\times$ & $\times$ & $\checkmark$ \\
\hline Different poses & $\times$ & $\times$ & $\checkmark$ \\
\hline $\begin{array}{c}\text { Processing } \\
\text { time }\end{array}$ & Low & Low & High \\
\hline
\end{tabular}

Result for neural network with different pose is as shown below.

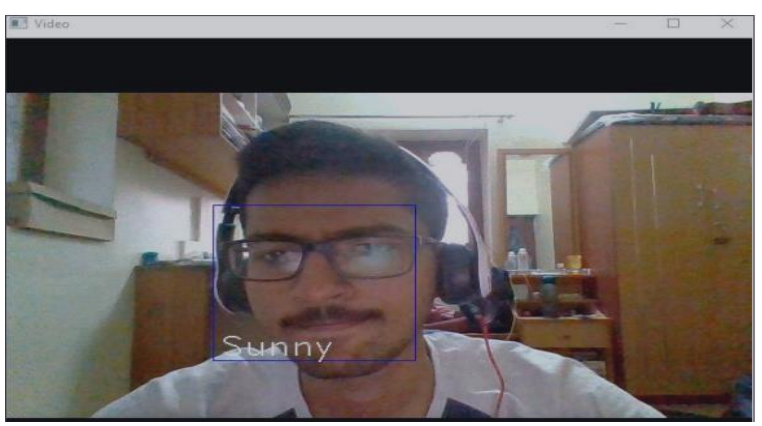

Result for neural network at different angles is as shown below.

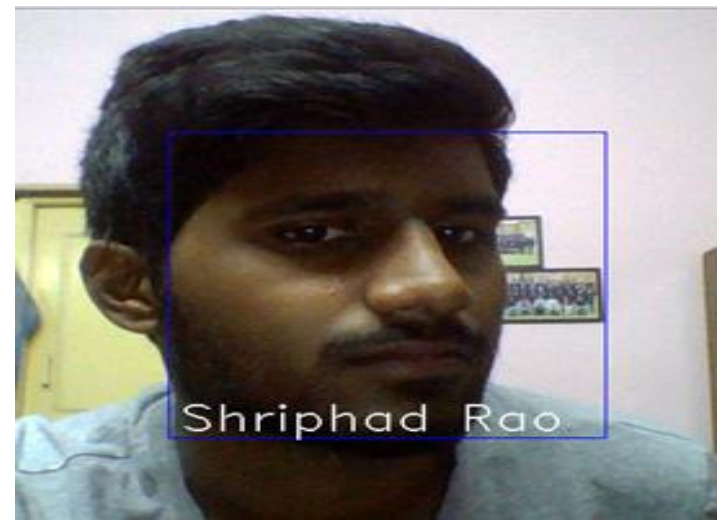

\section{FUTURE SCOPE}

The eventual fate of face recognition innovation is splendid. Forecasters opine that this innovation is relied upon to develop at an imposing rate and will create tremendous incomes in the coming years. Security and reconnaissance are the significant fragments which will be profoundly impacted. Different zones that are currently greeting it wholeheartedly are private ventures, open structures, and schools. It is evaluated that it will likewise be received by retailers and banking frameworks in coming a long time to keep misrepresentation in charge/Visa buys and installment particularly the ones that are on the web. This innovation would fill in the escape clauses of to a great extent common deficient secret phrase framework. Over the long haul, robots utilizing face recognition innovation may likewise come. They can be useful in finishing the undertakings that are illogical or hard for individuals to finish.

Although Deep learning has brightest scope of all technologies, even NVIDIA has increased the lifespan of deep learning by creating more CUDA. So, in future processing time will be a lot less compared to now.

\section{CONCLUSION}

This uses face recognition to display the proposed system door access system. The Home Door Lock control system has been successfully tested control by verifying the nominal face images based on the face recognition method. The proposed system is fully stand-alone and wireless to create a safe network that is reliable, stable, easy to operate and low cost. To successfully develop this entire system as a standalone security device, a battery power source has been installed. Finally this study concludes that the different operations have been checked successfully and the findings have been reported. 


\section{REFERENCES}

1. Hteik Htar Lwin, Aung Soe Khaing, Hla Myo Tun, “Automatic Door Access System UsingFace Recognition”, International Journal of Scientific \& Technology Research Volume 4, Issue 06, June 2015.

2. Sadeque Reza Khan, Ahmed Al Mansur, Alvir Kabir, Shahid Jaman, Nahian Chowdhury, "Design and Implementation of Low-Cost Home Security System using GSM Network", International Journal of Scientific \& Engineering Research, Volume 3Issue 3, March 2012.

3. Shambhavi V. Chippa, Dr. R. R. Dube, 2019, AWS EC2 based Home Security System using Face.

4. Oleksii Kharkovyna, An Intro to Deep Learning for FaceRecognition: https://towardsdatascience.com/an-intro-to-deep-learning-for-face-re cognition-aa8dfbbc51fb

5. Delbiaggio, N., 2017. A comparison of facial recognition's algorithms.

6. M. Turk and A. Pentland,"Face recognition using eigenfaces," in 2013 IEEE Conference on Computer Vision and Pattern Recognition, Maui, HI, USA, 1991 pp. 586,587,588,589,590,591.

7. ErikHjelmås, Boon KeeLow, Computer Vision and Image Understanding, Volume 83, Issue 3, September 2001, Pages 236-274

8. Belhumeur, P.N., Hespanha, J.P. and Kriegman, D.J., 1997. Eigenfaces vs. fisherfaces: Recognition using class specific linear projection. IEEE Transactions on Pattern Analysis \& Machine Intelligence, (7), pp.711-720.

9. LeCun, Y., Bengio, Y. and Hinton, G., 2015. Deep learning. nature, 521(7553), pp.436-444

10. Krizhevsky, A., Sutskever, I. and Hinton, G.E., 2012. Imagenet classification with deep convolutional neural networks. In Advances in neural information processing systems (pp. 1097-1105).

11. He, K., Zhang, X., Ren, S. and Sun, J., Deep Residual Learning for Image Recognition.

12. King, D. E, Dlib-ml: A Machine learning Toolkit. Journal of Machine Learning Research, pp. 1755-1758

13. Shehzad Noor Taus Priyo, Facial Recognition Using Deep Learning: https://towardsdatascience.com/facial-recognition-using-deep-learnin g-a74e9059a150

14. Geitgey, A. and Nazario, J., 2017. Face Recognition. En ligne] Disponible sur: https://github.com/ageitgey/face_recognition

15. https://www.electroschematics.com/nodemcu-iot-guide/

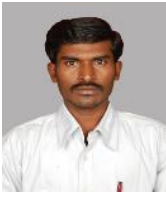

\section{AUTHORS PROFILE}

Mr.Telugu Maddileti is currently working as Assistant Professor in the department of Electronics and Computer at SNIST and Research Scholar at JNTUA, Ananthapuramu. He has a total of 14 years experience in academics. He completed B.Tech degree (Electronics and communication Engineering) in the year 2005 and M.Tech in 2009 from JNT University, Hyderabad. He is enthusiastic to work in the field of VLSI and Image Processing. His area of interest includes Low power VLSI domain, optimization of analog and mixed signal circuits in addition to digital system design using CAD tools. He has presented more than 15 International/National Technical Papers in journals \& conferences.

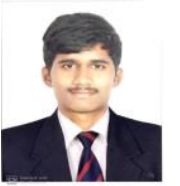

G. Shriphad Rao pursuing his B.Tech degree from JNTU, Hyderabad. His areas of interest includes communications, image processing and IOT based designs and applications. He has Presented 4 International/National Technical Papers in journals \& conferences

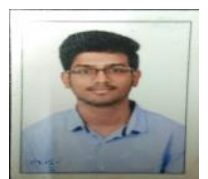

Vaddemani Sai Madhav pursuing his B.Tech degree from JNTU, Hyderabad. His areas of interest includes communications, image processing and IOT based designs and applications. He has Presented 4 International/National Technical Papers in journals \& conferences.

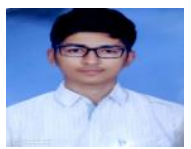

Ghanti Sharan pursuing his B.Tech degree from JNTU, Hyderabad. His areas of interest includes communications, image processing and IOT based designs and applications. He has Presented 4 International/National Technical Papers in journals \& conferences. 